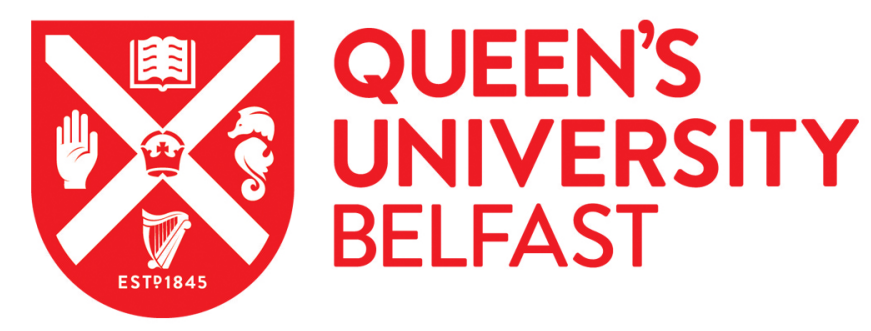

\title{
Quantum conductance of multiwall carbon nanotubes
}

Yoon, Y-G., Delaney, P., \& Louie, S. G. (2002). Quantum conductance of multiwall carbon nanotubes. Physical Review B (Condensed Matter), 66(7), 073407-1-073407-4. [073407].

https://doi.org/10.1103/PhysRevB.66.073407

Published in:

Physical Review B (Condensed Matter)

Queen's University Belfast - Research Portal:

Link to publication record in Queen's University Belfast Research Portal

\section{General rights}

Copyright for the publications made accessible via the Queen's University Belfast Research Portal is retained by the author(s) and / or other copyright owners and it is a condition of accessing these publications that users recognise and abide by the legal requirements associated with these rights.

Take down policy

The Research Portal is Queen's institutional repository that provides access to Queen's research output. Every effort has been made to ensure that content in the Research Portal does not infringe any person's rights, or applicable UK laws. If you discover content in the Research Portal that you believe breaches copyright or violates any law, please contact openaccess@qub.ac.uk. 


\title{
Quantum conductance of multiwall carbon nanotubes
}

\author{
Young-Gui Yoon,* Paul Delaney, and Steven G. Louie \\ Department of Physics, University of California at Berkeley, Berkeley, California 94720 \\ and Materials Sciences Division, Lawrence Berkeley National Laboratory, Berkeley, California 94720
}

(Received 5 April 2002; published 2 August 2002)

\begin{abstract}
An analysis on the conductance of multiwall carbon nanotubes (MWNT's) is presented. Recent experiment indicated that MWNT's are good quantum conductors. Our theory shows that tunneling current between states on different walls of a defect-free, infinitely long MWNT is vanishingly small in general, which leads to the quantization of the conductance of the MWNT's. With a reasonable simple model, we explicitly show that the conductance of a capped MWNT can be determined by the outermost wall for an infinitely long nanotube. We apply the theory to finite MWNT's and estimate the generic interwall conductance to be negligible compared to the intrawall ballistic conductance.
\end{abstract}

DOI: 10.1103/PhysRevB.66.073407

PACS number(s): 72.80.Rj, 81.05.Tp

\section{INTRODUCTION}

Carbon nanotubes are one of the most interesting new materials discovered in recent years. ${ }^{1}$ Considerable theoretical and experimental effort has already been focused on predicting and measuring the properties of various classes of tubes-multiwall nanotubes (MWNTs), singlewall nanotubes (SWNTs), and ropes of nanotubes. ${ }^{2}$ These materials promise exceptional mechanical strength and unusual electronic properties, hence motivating a great deal of investigation into their use as nanoscale devices. ${ }^{3-13}$ In this paper we investigate the physics of quantum conductance in the SWNT's and MWNT's. For a perfect SWNT with $n$ band crossing the Fermi level, a naive application of Landauer formula ${ }^{14,15}$ within the one-electron picture would yield a ballistic conductance of $n G_{0}$, where $G_{0}=2 e^{2} / h$ is the quantum unit of conductance. Extending this argument to a MWNT would lead to a ballistic conductance of $G=\Sigma_{i} G_{i}$, where $G_{i}$ is the conductance of the $i$ th wall, if all the walls participate in the transport of electrons. ${ }^{15}$ However, for MWNT's with closed caps, recent measurements ${ }^{19}$ have indicated that the conductance for such systems is quantized to the value of $G=G_{0}$. Thus, it appears that effectively only one channel is available for conduction in these multiwall systems, even though most metallic single-wall carbon nanotubes have two bands crossing the Fermi level.

In this work, we argue that this rather surprising finding may be explained by the blocked interwall tunneling current between the states of different walls and that, effectively, only the outermost wall is active in the electron transport when current is injected to the body of a capped MWNT. We first consider a two-wall carbon nanotube. Once we establish the behavior for the two-wall tube, the generalization to multiwall tubes is straightforward. Let the unit cell lengths along the inner and outer tube axes be $a_{\text {in }}$ and $a_{\text {out }}$, respectively. Two distinct cases naturally arise: the structure is either commensurate $\left(a_{\text {in }} / a_{\text {out }}\right.$ is rational $)$ or incommensurate $\left(a_{\text {in }} / a_{\text {out }}\right.$ is irrational). The first case is easier to dispose of and it illustrates the second case, so we discuss it first. As a concrete example, we may consider a two-wall tube with both walls of $(n, n)$ type. ${ }^{16-18} \mathrm{~A}$ schematic band structure of this two-wall tube is shown in Fig. 1. Tunneling of an electron in an unperturbed eigenstate on one wall to one on the other must obey both energy conservation and Bloch wave vector conservation, as the complete structure has a well-defined periodicity in the axial direction. As can be seen from Fig. 1, for general position of the Fermi level, it is impossible to tunnel between two states at the Fermi level and conserve tube crystal momentum. Thus, for an infinitely long commensurate MWNT, any overlap matrix element $\left\langle\psi_{\text {in }}|V| \psi_{\text {out }}\right\rangle$ which would tend to mix the two states at $E_{F}$ will be exactly zero. In this case, if a closed MWNT is immersed in mercury to complete an electrical circuit as described in Ref. 19, only the outermost wall's electronic states will couple significantly to the liquid metal and so the conductance measured will be that relevant to just the outermost wall. In the case that energy bands are nearly degenerate at very close $k$ vectors, however, interwall interaction can lead to eigenstates with measurable weight at inner walls. ${ }^{20}$ It is also possible for tiny gaps or pseudogaps to form at the band crossing points due to the interaction. In practice, the Fermi level is unlikely to be located exactly at the tiny gap region, and the electrical conduction is not affected by the formation of gaps or pseudogaps.

\section{THEORETICAL ANALYSIS}

The analysis of the incommensurate MWNT is more involved. It is not possible to use conservation of crystal mo-

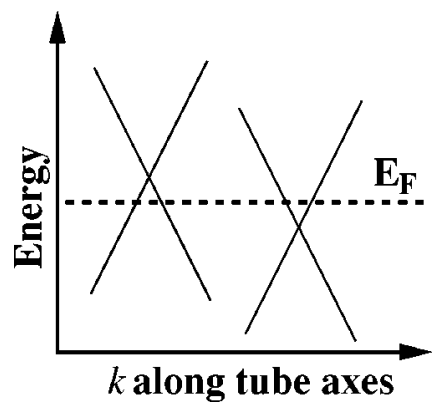

FIG. 1. Schematic band structure of a two-wall MWNT in positive $k$ direction. Due to different curvature effects, the position of the band crossing shifts to different values. 
mentum as an exact argument to dispose of any tunneling between states on different walls at $E_{F}$, as our complete structure does not have long-range periodicity, and hence, no crystal momentum can be defined for the structure as a whole. To investigate the way tunneling occurs among these states, we adopt Bardeen's tunneling Hamiltonian formalism. ${ }^{21}$ We demonstrate that there is a selection rule for transition between Bloch states of adjacent walls of an infinite MWNT. Using this simple but reasonable model, we also give an estimate of how well this selection rule holds for finite length MWNT's.

Bardeen's formalism ${ }^{21}$ in calculating tunneling current to first order for small voltage and temperature is

$$
\begin{gathered}
I=\frac{2 \pi e^{2}}{\hbar} V \sum_{\mu \nu}\left|M_{\mu \nu}\right|^{2} \delta\left(E_{\mu}-E_{F}\right) \delta\left(E_{\nu}-E_{F}\right), \\
M_{\mu \nu}=\frac{\hbar^{2}}{2 m_{e}} \int d \vec{S} \cdot\left(\psi_{\mu}^{*} \vec{\nabla} \psi_{\nu}-\psi_{\nu} \vec{\nabla} \psi_{\mu}^{*}\right) .
\end{gathered}
$$

The integral is over any surface region within the tunneling barrier. The essential part of the problem is in evaluating the matrix element $M_{\mu \nu}$. To do this, we expand the wave functions of each isolated wall of the MWNT in terms of localized orbitals in the tight binding approximation or more generally in terms of Wannier functions. Then by suitable rearrangement of terms, we can reproduce the $k$ selection rule of commensurate MWNT's and get similar selection rule for infinite length incommensurate MWNT's. For finite length MWNT's, we modeled the overlap integral needed for the matrix element calculation and estimated the corresponding conductance. We note at this point that, because of the overall cylindrical symmetry of the MWNT's, if $\psi_{\text {out }}$ and $\psi_{\text {in }}$ are of different rotational symmetry then there will not be any tunneling between the two states. However, we shall set this special case aside, and consider the more interesting general case.

We use in and out to index the inner and outer walls of different periodicity. The unperturbed individual wall states (with no interwall interaction) within a tight-binding model may be written as

$$
\begin{gathered}
\psi\left(\vec{k}_{\mathrm{in}}, \vec{r}\right)=\frac{1}{\sqrt{N_{\text {in }}}} \sum_{\vec{R}_{\text {in }}} e^{i \vec{k}_{\text {in }} \cdot \vec{R}_{\text {in }}} \phi_{\text {in }}\left(\vec{r}-\vec{R}_{\text {in }}\right), \\
\psi\left(\vec{k}_{\text {out }}, \vec{r}\right)=\frac{1}{\sqrt{N_{\text {out }}}} \sum_{\vec{R}_{\text {out }}} e^{i \vec{k}_{\text {out }} \cdot \vec{R}_{\text {out }}} \phi_{\text {out }}\left(\vec{r}-\vec{R}_{\text {out }}\right) .
\end{gathered}
$$

To make the analysis simple, we introduce the following quantities:

$$
\begin{gathered}
f\left(\vec{R}_{\text {out }}-\vec{R}_{\text {in }}\right)=\int d \vec{S} \cdot \phi_{\text {in }}^{*}\left(\vec{r}-\vec{R}_{\text {in }}\right) \vec{\nabla} \phi_{\text {out }}\left(\vec{r}-\vec{R}_{\text {out }}\right), \\
\Gamma\left(\vec{k}_{\text {out }}, \vec{R}_{\text {in }}\right)=\sum_{\vec{R}_{\text {out }}} e^{i \vec{k}_{\text {out }} \cdot\left(\vec{R}_{\text {out }}-\vec{R}_{\text {in }}\right)} f\left(\vec{R}_{\text {out }}-\vec{R}_{\text {in }}\right),
\end{gathered}
$$

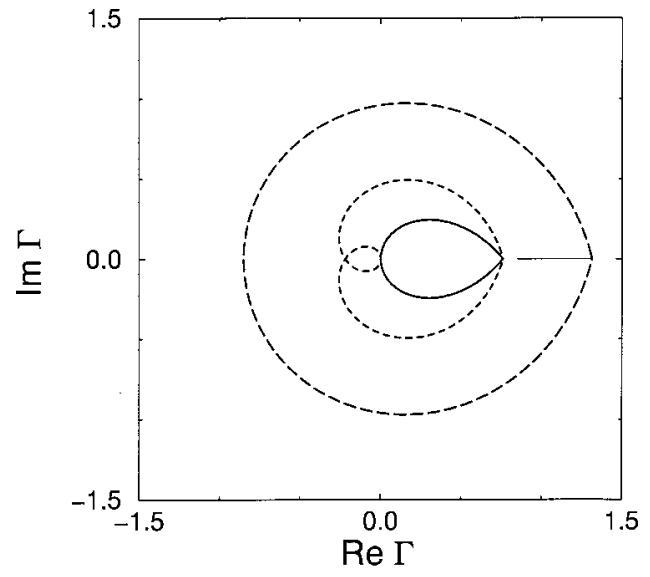

FIG. 2. $\Gamma\left(\vec{k}_{\text {out }}, \vec{R}_{\text {in }}\right)$ in complex plane for small $k_{\text {out }}$ as given by Eq. (12). The thin solid line is for $k_{\text {out }}=0$, the thick solid curve is for $k_{\text {out }}=0.5 G_{\text {out }}$, the dash curve is for $k_{\text {out }}=G_{\text {out }}$, and the long dash curve is for $k_{\text {out }}=1.5 G_{\text {out }}$.

where $\vec{S}$ is a cylindrical surface midway between the inner and outer walls. Similarly we define $\widetilde{f}$ and $\widetilde{\Gamma}$ as the above expressions with $\phi_{\text {in }}^{*}$ and $\phi_{\text {out }}$ interchanged. With these definitions, we can rewrite the tunneling matrix element as

$$
\begin{aligned}
M\left(\vec{k}_{\text {in }}, \vec{k}_{\text {out }}\right)= & \frac{1}{\sqrt{N_{\text {in }} N_{\text {out }}}} \frac{\hbar^{2}}{2 m} \sum_{\vec{R}_{\text {in }}} e^{i\left(\vec{k}_{\text {out }}-\vec{k}_{\text {in }}\right) \cdot \vec{R}_{\text {in }}\left\{\Gamma\left(\vec{k}_{\text {out }}, \vec{R}_{\text {in }}\right)\right.} \\
& \left.-\widetilde{\Gamma}\left(\vec{k}_{\text {out }}, \vec{R}_{\text {in }}\right)\right\}
\end{aligned}
$$

We note at this point that, from the form of Eqs. (2)- (4), $M\left(\vec{k}_{\text {in }}, \vec{k}_{\text {out }}\right)=M\left(\vec{k}_{\text {in }}-n \vec{G}_{\text {in }}, \vec{k}_{\text {out }}-m \vec{G}_{\text {out }}\right)$, for any integer $n$ and $m .\left(\vec{G}_{\text {in }}\right.$ and $\vec{G}_{\text {out }}$ are the reciprocal primitive translation vector of the inner and outer tube, respectively.) We shall make use of this fact in our analysis below.

\section{INFINITE MWNT}

For a commensurate MWNT, $\Gamma\left(\vec{k}_{\text {out }}, \vec{R}_{\text {in }}\right)$ as a function of $\vec{R}_{\text {in }}$ can only take on a few discrete values for a given $\vec{k}_{\text {out }}$. This naturally gives rise to the selection rule for $\vec{k}$ vectors. For the incommensurate case, the problem is nontrivial. $\Gamma\left(\vec{k}_{\text {out }}, \vec{R}_{\text {in }}\right)$ for a specific $\vec{R}_{\text {in }}$ is determined by the relative position of the lattice $\left\{\vec{R}_{\text {out }}\right\}$ with respect to the given $\vec{R}_{\text {in }}$, or equivalently, the distance to the nearest lattice point $\vec{R}_{\text {out }}$ on the right side of this particular $\vec{R}_{\text {in }}$ which takes on the continuous value from 0 to one lattice constant $a_{\text {out }}$ of the outer tube. The values of $\Gamma\left(\vec{k}_{\text {out }}, \vec{R}_{\text {in }}\right)$ for different $\vec{R}_{\text {in }}$ 's is then a collection of bounded complex numbers. (See Figs. 2 and 3.) Unless $\vec{k}_{\text {in }}=\vec{k}_{\text {out }}$ or the special case of $\vec{k}_{\text {in }}-n \vec{G}_{\text {in }}=\vec{k}_{\text {out }}$ $-m \vec{G}_{\text {out }}$, for some integer $m$ and $n$,

$$
\frac{1}{\sqrt{N_{\text {in }} N_{\text {out }}}} \sum_{\vec{R}_{\text {in }}} e^{i\left(\vec{k}_{\text {out }}-\vec{k}_{\text {in }}\right) \cdot \vec{R}_{\text {in }} \Gamma\left(\vec{k}_{\text {out }}, \vec{R}_{\text {in }}\right)}
$$




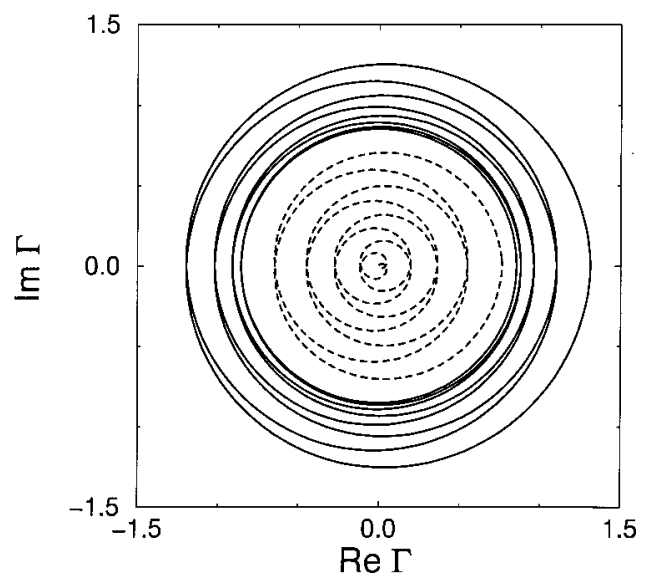

FIG. 3. $\Gamma\left(\vec{k}_{\text {out }}, \vec{R}_{\text {in }}\right)$ in complex plane for large $k_{\text {out }}$ as given by Eq. (12). The solid curve is for $k_{\text {out }}=8 G_{\text {out }}$, and the dash curve is for $k_{\text {out }}=8.5 G_{\text {out }}$.

is average over bounded fluctuating numbers and converges to zero for an infinite MWNT. The same argument holds for a general n-wall nanotube and establishes quasi crystal momentum conservation for long incommensurate MWNT's, where periodicity can be defined only for each wall individually. This selection rule blocks interwall tunneling current between the states of different walls at $E_{F}$.

\section{FINITE MWNT}

For a finite length MWNT, the sums in Eqs. (6) and (7) are over finite number of terms. Still, $\Gamma\left(\vec{k}_{\text {out }}, \vec{R}_{\text {in }}\right)$ can be defined to be an infinite sum with negligible error provided that $f$ decays exponentially and the length of the system is much larger than the characteristic decay length. As for the matrix element $M\left(\vec{k}_{\text {in }}, \vec{k}_{\text {out }}\right)$, we use the fact that $M\left(\vec{k}_{\text {in }}\right.$ $\left.-n \vec{G}_{\text {in }}, \vec{k}_{\text {out }}-m \vec{G}_{\text {out }}\right)=M\left(\vec{k}_{\text {in }}, \vec{k}_{\text {out }}\right)$. For a finite MWNT of length $L$, we can always find integer $m$ and $n$ such that $\left|\vec{k}_{\text {out }}-\vec{k}_{\text {in }}-\left(m \vec{G}_{\text {out }}-n \vec{G}_{\text {in }}\right)\right|<2 \pi / L$. The integers $m$ and $n$ should be restricted to values less than the number of unit cells in the tubes. Then the phase factor in Eq. (7) may be set to 1 and we can estimate the matrix element to be approximately

$$
\begin{aligned}
& M\left(\vec{k}_{\text {in }}, \vec{k}_{\text {out }}\right) \approx \frac{\hbar^{2}}{2 m_{e}} \frac{1}{N_{\text {in }}} \sum_{\vec{R}_{\text {in }}}\left\{\Gamma\left(\vec{k}_{\text {out }}-m \vec{G}_{\text {out }}, \vec{R}_{\text {in }}\right)\right. \\
& \left.-\widetilde{\Gamma}\left(\vec{k}_{\text {out }}-m \vec{G}_{\text {out }}, \vec{R}_{\text {in }}\right)\right\} \\
& \equiv \frac{\hbar^{2}}{2 m_{e}}\left\{\left\langle\Gamma\left(\vec{k}_{\text {out }}-m \vec{G}_{\text {out }}, \vec{R}_{\text {in }}\right)\right\rangle\right. \\
& \left.-\left\langle\widetilde{\Gamma}\left(\vec{k}_{\text {out }}-m \vec{G}_{\text {out }}, \vec{R}_{\text {in }}\right)\right\rangle\right\} .
\end{aligned}
$$

\section{SIMPLE MODEL}

We use the following simple model to calculate the quantity $\langle\Gamma\rangle$ explicitly. Assuming that

$$
f\left(\vec{R}_{\text {out }}-\vec{R}_{\text {in }}\right)=f_{0} e^{-\frac{\left|\vec{R}_{\text {out }}-\vec{R}_{\text {in }}\right|}{d}},
$$

where

$$
f_{0}=\int d \vec{S} \cdot \phi_{\text {in }}^{*}(\vec{r}) \vec{\nabla} \phi_{\text {out }}(\vec{r}) .
$$

In evaluating Eq. (6), let us denote the first lattice vector $\vec{R}_{\text {out }}$ on the right side of the particular $\vec{R}_{\text {in }}$ to be $\vec{R}_{\text {in }}+\vec{\alpha}$, and the primitive translation vector of the outer tube to be $\vec{a}_{\text {out }}$. Then

$$
\begin{aligned}
& \vec{R}_{\text {out }}-\vec{R}_{\text {in }}= \begin{cases}\vec{\alpha}+n \vec{a}_{\text {out }} & n=0,1,2, \cdots \\
-\left(-\vec{\alpha}+n^{\prime} \vec{a}_{\text {out }}\right) & n^{\prime}=1,2, \cdots\end{cases} \\
& \Gamma\left(\vec{k}_{\text {out }}, \vec{R}_{\text {in }}\right) \\
& =\sum_{\vec{R}_{\text {out }}} \mathrm{e}^{i \vec{k}_{\text {out }} \cdot\left(\vec{R}_{\text {out }}-\vec{R}_{\text {in }}\right)} f_{0} \mathrm{e}^{-\mid \vec{R}_{\text {out }}-\vec{R}_{\text {in }} / d} \\
& =\frac{f_{0} e^{i \vec{k}_{\text {out }} \cdot \vec{\alpha}}\left[e^{\left.-i \vec{k}_{\text {out }} \cdot a_{\text {out }} \sinh (\alpha / d)+\sinh \left(\left(a_{\text {out }}-\alpha\right) / d\right)\right]}\right.}{\cosh \left(a_{\text {out }} / d\right)-\cosh \vec{k}_{\text {out }} \cdot \vec{a}_{\text {out }}} .
\end{aligned}
$$

We show the value of $\Gamma$ as a function of $\vec{R}_{\text {in }}$ for some selected values of $k_{\text {out }}$ in Figs. 2 and 3.

For an estimate of $\langle\Gamma\rangle$, we may convert the average over many terms to an average over the continuous variable $\alpha$ assuming that $\alpha$ is uniformly distributed between 0 and $a_{\text {out }}$. Moreover, for any pair $\left(\vec{k}_{\text {in }}, \vec{k}_{\text {out }}\right)$, once we find $\mid\left(\vec{k}_{\text {out }}-\vec{k}_{\text {in }}\right.$ $-\left(m \vec{G}_{\text {out }}-n \vec{G}_{\text {in }}\right) \mid$ of the order of $2 \pi / L$, evaluation of $M\left(\vec{k}_{\text {in }}, \vec{k}_{\text {out }}\right)$ becomes a calculation of

$$
\begin{aligned}
\left\langle\Gamma\left(\vec{k}_{\text {out }}-m \vec{G}_{\text {out }}, \vec{R}_{\text {in }}\right)\right\rangle & \approx \frac{1}{a_{\text {out }}} \int_{0}^{a_{\text {out }}} \Gamma\left(\vec{k}_{\text {out }}-m \vec{G}_{\text {out }}, \vec{\alpha}\right) d \alpha \\
& =f_{0} \frac{d}{a_{\text {out }}} \frac{1}{1+\left(\left|\vec{k}_{\text {out }}-m \vec{G}_{\text {out }}\right| d\right)^{2}} .
\end{aligned}
$$

The number of $k$ states for a finite MWNT is of the order of $L / a_{\text {out }}$. Such a set of points, with average spacing of $2 \pi / L$, may be approximately generated by repeatedly translating vectors of length $l G_{\text {out }}$ (with $l=1, \ldots, L / a_{\text {out }}$ ) back to the first BZ defined by $G_{\text {in }}$ (i.e., $l G_{\text {out }}-k G_{\text {in }}$ ). Thus, for a given $k_{\text {in }}-k_{\text {out }}$, the most probable combination $m G_{\text {out }}-n G_{\text {in }}$ which gives $\mid\left(\vec{k}_{\text {out }}-\vec{k}_{\text {in }}-\left(m \vec{G}_{\text {out }}-n \vec{G}_{\text {in }}\right) \mid<2 \pi / L\right.$ would correspond to an $m$ in the order of $L / a_{\text {out }}$. If we take $m$ in Eq. (13) to be of the order of $L / a_{\text {out }}$, then we can readily estimate the average $\langle\Gamma\rangle$ to be the following:

$$
\langle\Gamma\rangle \approx \frac{f_{0}}{4 \pi^{2}} \frac{a_{\mathrm{out}}}{d}\left(\frac{a_{\mathrm{out}}}{L}\right)^{2} .
$$

This converges to zero very rapidly with increasing $L$. This is not surprising because the average of $\Gamma\left(\vec{k}_{\text {out }}-m \vec{G}_{\text {out }}, \vec{R}_{\text {in }}\right)$ should have the properties of the high spatial Fourier components of $f\left(\vec{R}_{\text {in }}-\vec{R}_{\text {out }}\right)$. The vanishingly small matrix elements for very long MWNT's are consistent with the quasi 
crystal momentum conservation for infinite MWNT's. Roughly, the longer the finite MWNT's, the better the selection rule is obeyed. We note that this argument is valid for weakly coupled walls in a MWNT.

So far, we only include the terms from $\psi_{\text {in }}^{*} \vec{\nabla} \psi_{\text {out }}$. Terms from $\psi_{\text {out }} \vec{\nabla} \psi_{\text {in }}^{*}$ are of the same order of magnitude. Thus, the most probable value for $M$ is

$$
M_{\text {in }, \text { out }} \approx A \frac{\hbar^{2}}{2 m_{e}} \frac{f_{0}}{4 \pi^{2}} \frac{a_{\text {out }}}{d}\left(\frac{a_{\text {out }}}{L}\right)^{2} .
$$

$A$ is a factor of order 1 and the interwall current $I_{\text {int }}$ is given by

$$
I_{\mathrm{int}}=\frac{2 \pi e^{2}}{\hbar} V\left|M_{\mathrm{in}, \mathrm{out}}\right|^{2} D_{\mathrm{in}}\left(E_{F}\right) D_{\mathrm{out}}\left(E_{F}\right)
$$

where $D\left(E_{F}\right)$ is the density of states at Fermi energy. To get a rough estimate, we put $d \approx a_{\text {out }}, f_{0} \approx 1 / a_{\text {out }}^{2}, D\left(E_{F}\right)$ $\approx L m_{e} a_{\text {out }} / \pi^{2} \hbar^{2}$ :

$$
G_{\text {int }}=\frac{I}{V} \approx \frac{2 e^{2}}{h}\left(\frac{a_{\text {out }}}{L}\right)^{2} .
$$

If we put $L=1 \mu \mathrm{m}, a_{\text {out }}=1 \AA$, we get

$$
G_{\text {int }} \approx 10^{-8} G_{0},
$$

and interwall conduction is then negligible.

In this analysis, We assume that electrons are injected from outside as eigenstates of the outermost wall at the Fermi level. If electrons are injected as very localized wave packets, the wave packet can be considered as linear combi- nation of eigenstates with broad range of Bloch wave vectors. The time evolution of the wave packet can lead to sizable penetration of electrons into inner walls, ${ }^{22}$ which is not surprising because the more localized the wave packet is, the more severely it lacks translational symmetry and the weaker the quasicrystal momentum conservation is. In that case, electrical conductance would be affected by the timedependent spread of the wave packet in the radial direction.

Similarly, MWNT's composed of perfect and incommensurate SWNT's will have only the outermost wall contributing to their STM images. However, if the translational symmetry of one of the constituent SWNT's is broken, interwall interaction may modify the outermost wall images.

\section{CONCLUSION}

In summary, we have presented an analysis of the quantum conductance of MWNT's, based on quasicrystal momentum conservation for the weakly coupled onedimensional system, using Bardeen's tunneling Hamiltonian formalism. Using a simple model we show that the conductance of a defect-free MWNT is dominated by the outermost wall, i.e., vanishingly small interwall conductance compared to intratube ballistic conductance for finite but reasonably long MWNT.

\section{ACKNOWLEDGMENTS}

This work was supported by National Science Foundation Grant No. DMR00-87088, and by the Director, Office of Science, Office of Basic Energy Sciences, Division of Materials Sciences and Engineering, U.S. Department of Energy under Contract No. DE-AC03-76SF00098.
*Current address: Princeton Materials Institute, Princeton University, Princeton, NJ 08544.

${ }^{1}$ S. Ijima, Nature (London) 354, 56 (1991).

${ }^{2}$ S. G. Louie, in Proceedings of Welch Foundation 40th Conference on Chemical Research: Chemistry in the Nanometer Scale (The Robert A. Welch Foundation, Houston, 1998); Y.-G. Yoon and S. G. Louie, in Nanostructured Carbon for Advanced Applications, edited by G. Genedek, P. Milani, and V. G. Ralchenko (Kluwer Academic publishers, Dordrecht, 2001).

${ }^{3}$ M. Bockrath, D.H. Cobden, P.L. McEuen, N.G. Chopra, A. Zettl, A. Thess, and R.E. Smalley, Science 275, 1922 (1997).

${ }^{4}$ S.J. Tans, M.H. Devoret, H. Dai, A. Thess, R.E. Smalley, L.J. Geerligs, and C. Dekker, Nature (London) 386, 474 (1997).

${ }^{5}$ P.G. Collins, A. Zettl, H. Bando, A. Thess, and R.E. Smalley, Science 278, 100 (1997).

${ }^{6}$ S.J. Tans, R.M. Verschueren, and C. Dekker, Nature (London) 393, 49 (1998).

${ }^{7}$ L. Chico, V.H. Crespi, L.X. Benedict, S.G. Louie, and M.L. Cohen, Phys. Rev. Lett. 76, 971 (1996).

${ }^{8}$ L. Chico, L.X. Benedict, S.G. Louie, and M.L. Cohen, Phys. Rev. B 54, 2600 (1996)

${ }^{9}$ M. Fuhrer, J. Nygard, L. Shih, M. Foreo, Y.-G. Yoon, M.S.C. Mazzoni, H.J. Choi, J. Ihm, S.G. Louie, A. Zettl, and P.L. McEuen, Science 288, 494 (2000).
${ }^{10}$ Y.-G. Yoon, M.S.C. Mazzoni, H.J. Choi, J. Ihm, and S.G. Louie, Phys. Rev. Lett. 86, 688 (2001).

${ }^{11}$ H. Choi, J. Ihm, Y.-G. Yoon, and S.G. Louie, Phys. Rev. B 60, R14 009 (1999).

${ }^{12}$ S. Sanvito, Y.-K. Kwon, D. Tománek, and C.J. Lambert, Phys. Rev. Lett. 84, 1974 (1999).

${ }^{13}$ P. Delaney, M. Di Ventra, and S.T. Pantelides, Appl. Phys. Lett. 75, 3787 (1999).

${ }^{14}$ R. Landauer, IBM J. Res. Dev. 1, 223 (1957); R. Landauer, ibid. 32, 306 (1988).

${ }^{15}$ D.S. Fisher and P.A. Lee, Phys. Rev. B 23, 6851 (1981).

${ }^{16}$ J.W. Mintmire, B.I. Dunlap, and C.T. White, Phys. Rev. Lett. 68, 631 (1992)

${ }^{17}$ N. Hamada, S.-I. Sawada, and A. Oshiyama, Phys. Rev. Lett. 68, 1579 (1992).

${ }^{18}$ R. Saito, M. Fujita, G. Dresselhaus, and M.S. Dresselhaus, Appl. Phys. Lett. 60, 2204 (1992).

${ }^{19}$ S. Fank, P. Poncharal, Z.L. Wang, and W.A. de Heer, Science 280, 1744 (1998).

${ }^{20}$ Y.-K. Kwon and D. Tománek, Phys. Rev. B 58, R16 001 (1998).

${ }^{21}$ J. Bardeen, Phys. Rev. Lett. 6, 57 (1961).

${ }^{22}$ S. Roche, F. Triozon, A. Rubio, and D. Mayou, Phys. Rev. B 64, 121401 (2001) 